\title{
Theme: Hematology
}

\section{丹} Serum ferritin for the diagnosis of cardiac and liver iron overload in thalassemia (Br J Haematol. 2017 May 23. doi: 10.1111/bjh.14776. [Epub ahead of print])

The aim of this study from 8 centers across Thailand was to assess the accuracy of serum ferritin in the diagnosis of iron overload compared to magnetic resonance imaging (MRI) in patients with thalassemia for assessment of cardiac iron overload (CIO) and liver iron overload (LIO). A total of 405 patients were included with a mean (SD) age of 18.8 (12.5) years; $296(73 \%)$ had transfusion-dependent thalassemia, $61(15 \%)$ were not transfusion-dependent, and $48(12 \%)$ were non-transfused. CIO and LIO, respectively, were detected by MRI in 5\% and $57 \%$ of cases. Sensitivity of serum ferritin for CIO was excellent, but specificity was relatively low. The overall positive predictive value was $<10 \%$, indicating that serum ferritin was not appropriate for diagnosing CIO, but was suitable for excluding CIO. For LIO, sensitivity of serum ferritin was lower than for CIO, but specificity was better, indicating that serum ferritin can be used to diagnose LIO. The diagnostic accuracy of serum ferritin was sufficiently high to lead to a more efficient use of MRI resources. In regions with limited access to MRI, serum ferritin levels may be primarily used to rule out CIO and to rule in/diagnose LIO.

Low-dose ferrous sulfate vs iron polysaccharide complex for treatment of nutritional iron-deficiency anemia (JAMA. 2017;317:2297-304)

The aim of this randomized controlled trial was to compare the effect of ferrous sulfate with iron polysaccharide complex on hemoglobin concentration in infants and children with nutritional iron deficiency anemia (IDA) at a US tertiary care hospital. Children were administered $3 \mathrm{mg} / \mathrm{kg}$ of elemental iron once daily as either ferrous sulfate drops or iron polysaccharide complex drops for 12 weeks. Of 80 randomized infants and children, 59 completed the trial. From baseline to 12 weeks, mean hemoglobin increased from 7.9 to $11.9 \mathrm{~g} / \mathrm{dL}$ (ferrous sulfate group) $v s 7.7$ to $11.1 \mathrm{~g} / \mathrm{dL}$ (iron polysaccharide complex group), a greater difference of $1.0 \mathrm{~g} / \mathrm{dL}(P<.001)$ with ferrous sulphate. Among infants and children aged 9 to 48 months with IDA, ferrous sulphate compared with iron polysaccharide complex resulted in a greater increase in hemoglobin concentration at 12 weeks. Authors concluded that once daily ferrous sulfate should be considered for children with nutritional iron-deficiency anemia.

Another trial that reassures us to continue our trust with the economical, though 'non-glamorous', ferrous sulphate for IDA in our daily practice.

\section{Iron deficiency and Iron deficiency anemia in breastfed healthy infants (Indian J Pediatr. 2017;84:505-8).}

There is often a dilemma regarding the age of starting iron supplementation in healthy babies. The aim of this study from Chandigarh, India, was to assess the prevalence of iron deficiency (ID) and iron deficiency anemia (IDA) in predominantly breastfed, 3-5-mo-old infants born at term with a birth weight e" $2.5 \mathrm{~kg}$. Among 215 infants enrolled, the prevalence of ID at 3, 4 and 5 mo of age was $5.4 \%, 21.4 \%$ and $36.4 \%$, while that of IDA was $4.6 \%, 16.7 \%$ and $11.4 \%$, respectively. Given the adverse neuro-cognitive and developmental outcomes of ID in the developing brain, a prevalence of ID of $21.4 \%$ and $36.4 \%$ at the age of 4 and $5 \mathrm{mo}$, respectively, is concerning.

It is crucial to run similar studies with a larger sample size in different regions of India. If similar results are observed, it would be reasonable to recommend iron supplementation at 4 months (as already recommended by American Academy of Pediatrics in the USA), instead of the current practice of 6 months, recommended by National Iron plus Initiative in India, in predominantly breastfed 'healthy babies'.

Episodic replacement of clotting factor concentrates does not prevent bleeding or musculoskeletal damage in haemophilia (Haemophilia. 2017; 23:538-46)

A longitudinal study was carried out in 255 children from 10 centers in nine developing countries over 5 years to assess the musculoskeletal outcome of children on episodic factor replacement. The median (IQR) age at the beginning of the study was $10(7,12)$ years. The study demonstrated that with episodic factor replacement, there was no meaningful reduction in the frequency of bleeding. Patients were still likely to have significant joint disease by the time they reach their teens or early adulthood. Authors concluded that episodic treatment with clotting factor concentrates should not be recommended as a modality of long-term management of hemophilia. The critical implication of the data was that those patients in emerging countries who have access to $>1000 \mathrm{IU} / \mathrm{kg} /$ year of clotting factors should be immediately shifted to a prophylaxis regimen of at least 10-15 IU/kg twice a week. Authors concluded that prophylaxis is the only way to preserve musculoskeletal function in hemophilia.

A study with significant implications for decision makers of national health policy, and for distribution of resources.

DEEPAK BANSAL deepakbansaldr@gmail.com 